\title{
Problems in abundance determination from UV spectra of hot supergiants
}

\author{
M. Sarta Deković ${ }^{1}$, D. Kotnik-Karuza ${ }^{1}$, T. Jurkić ${ }^{1}$ \\ and D. Dominis Prester ${ }^{1}$ \\ ${ }^{1}$ Department of Physics, University of Rijeka, \\ Omladinska 14, 51000 Rijeka, Croatia \\ email: msarta@phy.uniri.hr
}

\begin{abstract}
We present measurements of equivalent widths of the UV, presumably photospheric lines: C III $1247 \AA$, N III $1748 \AA$, N III $1752 \AA$, N IV $1718 \AA$ and He II $1640 \AA$ in highresolution IUE spectra of 24 galactic OB supergiants. Equivalent widths measured from the observed spectra have been compared with their counterparts in the Tlusty NLTE synthetic spectra. We discuss possibilities of static plan-parallel model to reproduce observed UV spectra of hot massive stars and possible reasons why observations differ from the model so much.
\end{abstract}

Keywords. stars: abundances, stars: supergiants, ultraviolet: stars.

\section{Observation material}

High-resolution UV spectra of 24 galactic OB supergiants were obtained with IUE in the wavelength range 1200-1900 A. In this work we have selected the following nonresonance absorption photospheric lines: C III $1247 \AA$, N IV $1718 \AA$, N III $1748 \AA$, N III $1752 \AA$ and He II $1640 \AA$. Sample stars: HD 207198, HD 30614, HD 209975, HD 167264, HD 37128, HD 204172, HD 38771, HD 192422, HD 213087, HD 2905, HD 13854, HD 24398, HD 193183, HD 13841, HD 190603, HD 14818, HD 206165, HD 41117, HD 42087, HD 198478, HD 53138, HD 225094, HD 164353, HD 58350. Spectral type and effective temperatures are adopted from McErlean, Lennon \& Dufton (1999).

\section{Methods and discussion}

The aim of this work was to study the UV spectra of the hot massive stars and to find out their reliability in determination of abundances. We also wanted to investigate whether the present hydrostatic NLTE models could adequately reproduce the non-resonant UV absorption lines in the spectra of the B supergiants. Synthetic spectra were calculated with metal line-blanketed, NLTE, plane-parallel, hydrostatic code TLUSTY and SYNSPEC (Lanz \& Hubeny 2007, Lanz \& Hubeny 2003). The $\log g$ values are associated to $T_{\text {eff }}$ in agreement with Table 1. and Fig. 2 from McErlean, Lennon \& Dufton (1999). The synthetic spectra were convolved with the instrumental, rotational and macroturbulent broadening (Turnrose \& Thompson 1984, Cassatella \& Martin 1982, Dufton et al. 2006, Ryans et al. 2002). The selected spectral lines were broadened using the code ROTIN (http://nova.astro.umd.edu/Synspec43/synspec.html). In the first step we compared equivalent widths measured in the observed spectra with their counterparts from the synthetic spectra (Fig. 1) and found random deviations from the synthetic spectra, either processed or non-processed. This large discrepancy, even with the processed models, suggest that the appearance of high overabundance in $\mathrm{N}$ and He should be treated as a result of other effects: inevitable line blending in the UV spectra, especially 
in stars with large vsin $i$, which leads to overestimate of equivalent widths and underestimate of the continuum level. Moreover, the models do not include all lines that exist in real objects, the latter showing much stronger absorption features relative to the synthetic spectra. Further, the lines considered to be photospheric might be possible affected by neighboring resonant wind-contaminated line which are not included in hydrostatic Tlusty models. As the synthetic spectra were convolved with averaged rotational and macroturbulent velocities, large deviations for real objects may exist.
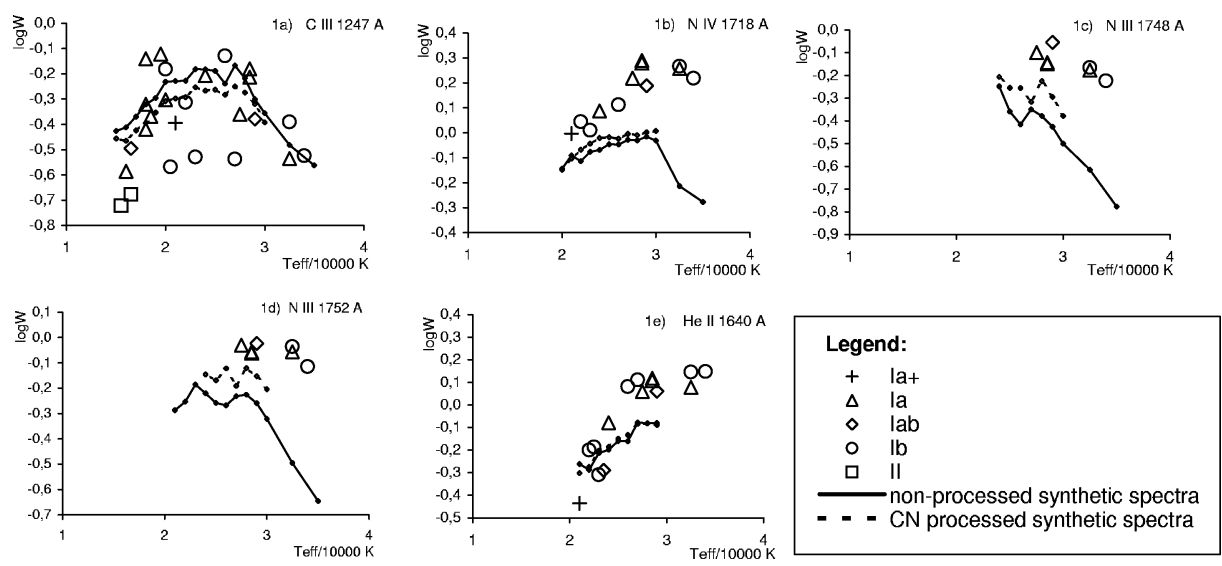

Figure 1. $\log W$ vs. $T_{\text {eff }}$ from observed (symbols) and synthetic (lines) spectra. The CN-processed model assumes a He abundance increased to $\mathrm{He} / \mathrm{H}=0.2$ by number, $\mathrm{N}$ abundance increased by a factor of 5 , and a halved $\mathrm{C}$ abundance.

\section{Conclusion}

Differential analysis is not sufficiently reliable in determination of abundances from UV spectra of B supergiants. In order to estimate the contribution of individual lines in the observed profile, exact modeling by use of adequate codes is needed. It is a question whether the present models are able at all to correctly produce strong UV lines. For example, the wind model CMFGEN has failed in this attempt (Searle et al. 2008). The non-wind Tlusty code models purely photospheric lines and can be successfully applied on weak optical lines. We have shown that use of Tlusty grids and convolution with rotational and macroturbulent broadening cannot reproduce the photospheric UV lines appropriately.

\section{References}

Cassatella, A. \& Martin, T. 1982, Report to IUE Three-Agency Coordinatiom Meeting

Dufton, P. L., Ryans, R. S. I., Simon-Diaz, S., Trundle, C., \& Lennon, D. J. 2006, A \& A, 451, 603

Lanz, T. \& Hubeny, I. 2007, ApJS, 169, 83

Lanz, T. \& Hubeny, I. 2003, ApJS, 146, 417

McErlean, N.D., Lennon, D.J., \& Dufton, P.L. 1999, A \& A, 349, 553

Ryans, R. S. I., Dufton, P. L., Rolleston, W. J. R., Lennon, D. J., Keenan, F. P., Smoker, V. J., \& Lambert, D. J. 2002, MNRAS, 336, 577

Searle, S. C., Prinja, R. P., Massa, D., \& Ryans, R. 2008, A \& A , 481, 777S

Turnrose, B. E. \& Thompson, R. W. 1984, Conctract NAS 5-27295 Task Assignment 401 